\title{
Dislocation emission caused by different types of nanoscale deformation defects in CdTe
}

\author{
V.N. Babentsov, V.A. Boyko, S.G. Gasan-zade, G.A. Shepelskii, S.V. Stariy \\ V. Lashkaryov Institute of Semiconductor Physics, NAS of Ukraine, \\ 41, prospect Nauky, 03028 Kyiv, Ukraine \\ Phone: 38 (044) 525-1813, e-mail: ssv1811@i.com.ua
}

\begin{abstract}
Dislocation-related defects induced by dislocation motion in p-CdTe were studied. Generation of "fresh" dislocations from the indented point of the CdTe (100), (110), and (111) surfaces at room temperatures was visualized by chemical etching and low temperature photoluminescence in a mapping regime. The crystallographic orientation of the dislocation rosettes of macroscopic plastic deformation lines was analyzed on the (100), (110), and (111) surfaces.
\end{abstract}

Keywords: photoluminescence, dislocation-related defect, dislocation emission.

Manuscript received 11.11.13; revised version received 22.01.14; accepted for publication 20.03.14; published online 31.03.14.

\section{Introduction}

Dislocation-related electronic states induced by dislocation motion in II-VI semiconductors are investigated for a long time due to their importance in solid state physics and for potential industrial applications [1-3].

Moving dislocations form various types of electronic states in the bandgap of semiconductors, namely, they may refer to different dislocations themselves, or to an impurity (native defect) segregated around dislocations. Different dislocation-related defects may form deep and shallow levels, stable and meta-stable, point and nanoscaled ones; they can vary depending on the particular type of dislocation-core dangling bonds, depending on the reconstructions of defects, and the strains around the dislocations, etc. However, it is not easy to show that the detected electronic states really originate from the dislocations themselves.

Among all representatives of the II-VI family of semiconductors, the cubic semi-insulated CdTe has attracted great attention of experts in physics and technology of various devices. Based on this fact, the objective of this contribution is to demonstrate some common features of the dislocation related states on the basis of this compound.
We have to underline, that the CdTe semiconductor belongs to semiconductors which are difficult to treat with due to their easy plasticity. Because of this, during the various standard technological operations that are necessary in the production of semiconductor devices, e.g., mechanical treatment, ion implantation, evaporation of various films, etc., a great number of dislocations and other defects of the deformation origin can be generated in the active volume of the device. They can be generated also in the operation period of the devices, such as lasers and photodiodes.

Earlier, we published results which concern experiments on the study deep and shallow energy levels introduced by dislocations in CdTe [4, 5], in this work we communicate the results of investigation of "fresh" dislocations on the (100), (110), and (111) surfaces of the $\mathrm{p}-\mathrm{CdTe}$ samples. These dislocations were introduced in CdTe by focused stress from the diamond indenter, and were studied with low temperature photoluminescence (PL) and chemical etching combined with the analysis of crystallographic orientation of the distributed dislocations.

As a result of the generation and movement of "fresh" dislocations from the indented point of the CdTe surface at room temperatures, followed by the chemical etching, a rosette of macroscopic plastic deformation lines appears on the surface. In the area of the arrows of 
a rosette the new emission lines appear in the low temperature PL spectrum of CdTe. These lines have no analogues in the PL spectrum of the original material, and, because of that, this recombination emission has been named as the "dislocation" emission. The "dislocation" PL normally consists of several lines, and is originated from the recombination on various complexes of defects that appear in the crystal lattice. Such complexes have very weak energies of dissociation, and they can be destroyed already at $T>$ $150 \ldots 200 \mathrm{~K}$.

Fig. 1 demonstrates the scheme of formation of onedimensional defect that was created due to dislocation movements. We can identify the dislocation type by comparing the profile of an intensity of the "dislocation" emission with crystallographic structure of lattice.

The aim of this work was to further clarify, on the basis of an analysis of the lattice deformation, either these defects belong to $\mathrm{Te}$ or $\mathrm{Cd}$ sublattices and to formulate a model of such defects.

\section{Experimental}

We investigated CdTe grown by the Bridgman Method from $6 \mathrm{~N}$ starting elements. The content of uncontrolled impurities in the grown crystals was about $10^{16} \mathrm{~cm}^{-3}$, as analyzed by glow-discharge mass spectrometry (GDMS). The oriented samples of $5 \times 8 \times 1 \mathrm{~mm}$ in dimensions were cut from the middle of the several ingots, mechanically lapped, polished, and finally chemo-mechanically polished with a Br/methanol solution. We applied a low-temperature photoluminescence (PL), to characterize the material's properties, and chemical etching with the Ag containing etchant to characterize the crystallographic orientation of dislocations on the (100) surface [6].

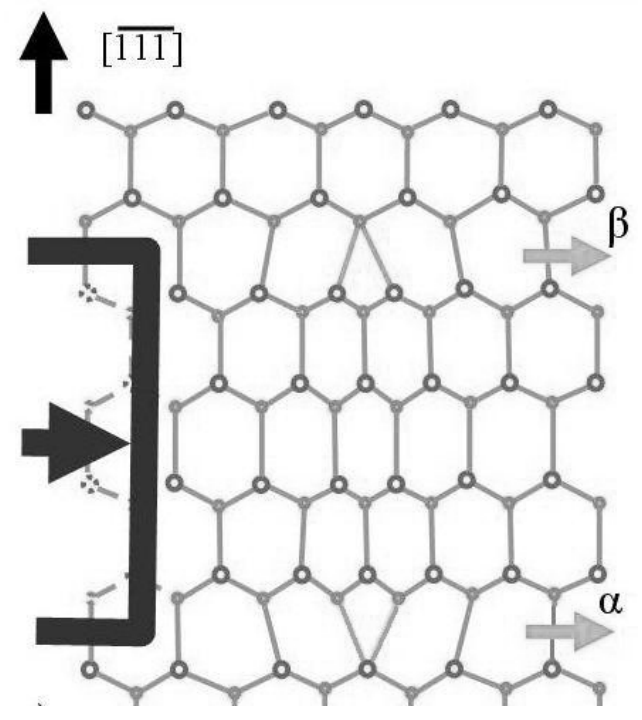

Fig. 1. Development of the glide (111) Te and Cd planes in the vicinity of deformation creates $\alpha$ and $\beta$ dispositions.

\section{Results and discussion}

Low-temperature photoluminescence. Fig. 2 (curve 1) demonstrates the PL spectrum measured at $4.2 \mathrm{~K}$ from the initial unindented (100) surface of the CdTe sample. This spectrum is a typical one for the $\mathrm{p}$ type CdTe, and it contains, respectively, the strong line of an acceptor bound exciton at $780 \mathrm{~nm}$ and a weak emission in the spectral region between 800 and $860 \mathrm{~nm}$, which is usually ascribed to the recombination on uncontrolled impurities. As it is shown in Fig. 2 (curves 2 and 3 ), the PL spectrum has drastically changed in the area of a fingerprint of an indenter: strong and structured emission bands appeared in the 800 to $860 \mathrm{~nm}$ spectral region being dependent on the excitation intensity. The most intensive PL band at $841 \mathrm{~nm}$ dominates the spectrum when an intensity of excitation is ten times higher than the excitation initially used for measurements on the untreated sample. Increased excitation leads to saturation of the 841-nm PL band, and a new PL band centered at $806 \mathrm{~nm}$ dominates the spectrum. As the saturation of the $841 \mathrm{~nm}$ line is clear evidenced with an increase of the excitation power, we conclude that such a type of defects either is in a minor concentration, or it has smaller cross-section for trapping of excitons.

A distinctive feature of the "dislocation" PL $(806 \mathrm{~nm})$ line is its instability: the band practically disappears in the PL spectra even after a relatively short (several hours) storage of indented crystals at room temperature. But, when we contain the indented crystal at lower temperatures $(T<100 \mathrm{~K})$, this band remains in the PL spectra for very long time (months). Another group of radiation bands 1.47 to $1.51 \mathrm{eV}(840 \ldots 820 \mathrm{~nm})$ is stable and remains in the spectra even after long storage of crystals at room temperature.

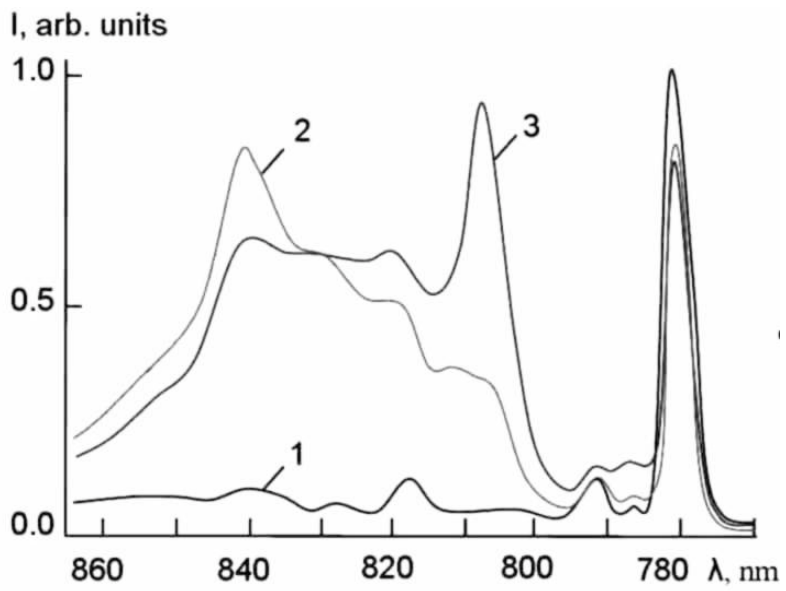

Fig. 2. The PL spectra from the (001) surface of the CdTe crystal at $4.2 \mathrm{~K}$ before $(1)$ and after indentation $(2,3)$. The curve 2 corresponds to the relation of the excitation intensities $I(2) / I(1)=10$, and the curve $3-$ to $I(3) / I(1)=500$, respectively. 
Temperature quenching of "dislocation" PL. The dependence of the "dislocation" related PL on temperature has been studied in order to understand its recombination properties. Fig. 3 demonstrates a typical temperature dependence of the PL spectra of the indented CdTe sample.

First, it has been found that, as it is known from previous investigations, the dislocation luminescence is rapidly quenched in the temperature interval of approximately $4.5 \ldots 100 \mathrm{~K}$. It was confirmed that, the PL line centred at $841 \mathrm{~nm}(1.47 \mathrm{eV})$, the so-called Y band, has a constant optical binding energy of $130 \mathrm{meV}$ at these temperatures. Two other stable "dislocation" bands, centred at 830 and $818 \mathrm{~nm}$, have a bit lower optical binding energies of 110 and $90 \mathrm{meV}$, respectively. Secondly, from this temperature dependence of the $\mathrm{Y}$ band intensity, the thermal activation energy $E_{A}$ for the quenching process could be determined. It appeared that the thermal activation energy for $841-\mathrm{nm}$ luminescence is determined to be about $10 \ldots 11 \mathrm{meV}$, and it is about $9 \mathrm{meV}$ for the 830 $\mathrm{nm}$ band, and $9 \mathrm{meV}$ for the 818 -nm band.

Following the ideas, that $841-\mathrm{nm}$ PL is originated from the recombination of excitons bound to extended defect related states, we can conclude that the 830- and 818-nm "dislocation" bands refer to recombination of less bounded excitons. The radiative recombination process is thus governed by the capture of free excitons to extended defects, and thermal quenching of the "dislocation" PL is determined by the thermal release of the bound excitons from the defect states.

The unstable "dislocation" emission centred at $10 \mathrm{~K}$ at $806 \mathrm{~nm}$ splits into two lines with the temperature increase, demonstrating the appearance of the higher wavelength component at $40 \ldots 60 \mathrm{~K}$. The origin of this splitting is not jet clear.

The spatial profile of the "dislocation emissions". We can identify the dislocation type by comparing the profile of an intensity of the "dislocation" emission with the crystallographic directions and the dislocation structure.

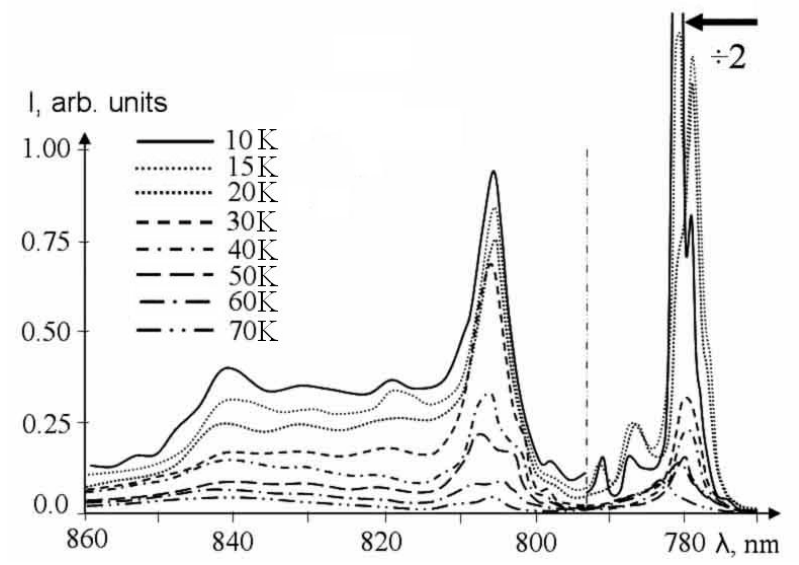

Fig. 3. Temperature dependence of the PL spectra of an indented CdTe crystal.
The arrows of a "rosette" are developed due to the glide of the principal dislocations in either $\mathrm{Cd}$ - or Teplanes. That is, the extra half-planes will be bounded only by $\mathrm{Cd}$ or $\mathrm{Te}$ atoms, respectively. In the case of an indentation of the (001) face, one pair of the symmetric arrows of the rosette moved from the indented point will be formed by segments of the 60-degree $\mathrm{Te}(\mathrm{g})$ dislocations (or, in other words, $\alpha$-dislocations), while the other pair of arrows will be formed by the segments of 60-degree $\mathrm{Cd}(\mathrm{g})$-dislocations (or $\beta$-dislocations).

As a result, in the case of indentation of the (001) face, conglomerates of the $\mathrm{Te}(\mathrm{g})$ - and $\mathrm{Cd}(\mathrm{g})$-dislocations appear to be spatially separated. This implies that the rows of indentation points on the (001) face should be in the perpendicular directions. Indeed, in this case, the regions adjacent to the rows of indentation points will contain on the crystal surface either only $\mathrm{Te}(\mathrm{g})$ dislocations when the indentation points are directed in the $\pm\langle 1 \overline{1} 0\rangle$ direction or only $\mathrm{Cd}(\mathrm{g})$-dislocations in the $\pm\langle 110\rangle$ direction.

That's why, to identify the defects, responsible for the appearance of the "dislocation" PL, an indentation of the (001) surface was performed exactly in the abovementioned directions. In such a geometry, one may expect a difference in the width of a spatial distribution of the intensities of the 820...840- and 806-nm PL bands, if they really associated with the $\mathrm{Te}(\mathrm{g})-$ or $\mathrm{Cd}(\mathrm{g})$ dislocations that moves for different distances from the indentation point.

Fig. 4 demonstrates that the spatial distributions of intensities of the 841 and 806-nm PL bands are really different. The half-widths of spatial profiles for 841-nm PL in two perpendicular directions differ by several times. At the same time, anisotropy in the profiles of the 806-nm band is practically absent.

Comparing the diagram of the dislocation structure with the fact that the half-width of the profile of the 841$\mathrm{nm}$ band for the $\pm\langle 1 \overline{1} 0\rangle$ direction exceeds that for the $\pm\langle 110\rangle$ direction by several times, one can make an unambiguous conclusion that the $841-\mathrm{nm}$ band is directly related to the electronic states of $\mathrm{Te}(\mathrm{g})$ dislocations. The width of the intensity profiles for the $820-$ and $830-\mathrm{nm}$ radiation bands has the same orientation dependence as that for the $841-\mathrm{nm}$ band. This testifies that all these PL bands can be related to the same type of defects, namely to Te(g)-dislocations.

The absence of a noticeable anisotropy of the radiation intensity profiles in the $806-\mathrm{nm}$ band evidences that this band cannot be related to the electronic states of either $\mathrm{Cd}(\mathrm{g})$ - or $\mathrm{Te}(\mathrm{g})$-dislocations. Taking into account also that the $806-\mathrm{nm}$ band is a metastable band, it is worth supposing that the 806-nm radiation cannot be directly related to the states of the dislocation core (as it turns out for the 841-nm band). It should be associated with the electronic states of less stable defects that arise due to the motion of dislocations. Moreover, the 
indicated defects also cannot be elementary point defects, as the 806- $\mathrm{nm}$ radiation in CdTe is observed only after the low temperature motion of dislocations. It doesn't arise due to any other influences on the crystal, which generate point defects (for example, due to the bombardment of the crystal with high-energy particles). The unstable states can be related to some ordered (complexes) structures of point defects that are formed as a result of motion of dislocations with steps, as it was shown earlier [1].

Fig. 4 contains also information that allows one to make a conclusion concerning the type of dislocations, which motion results in generation of the indicated metastable defects responsible for 806-nm radiation. Indeed, for the $\langle 110\rangle$ direction, the half-width of the profile of the 841-nm band appears to be much narrower than that of the 806-nm band profile. It's obvious that the point defects generated by mobile dislocations in no way can reach the part of the crystal, where the indicated dislocations couldn't get to. Such a statement is even more correct in the case of low temperatures. That's why one can conclude that the defects responsible for the 806-nm radiation should be associated with the structure of defects generated due to the glide of dislocation semiloops with principal $\mathrm{Cd}(\mathrm{g})$-dislocations. As is known, the motion of screw dislocations with steps represents an effective mechanism of formation of point defects. If this screw dislocation moves, the creep of a step along the way of its motion results in the generation of the nano-structures chains of point defects: vacancies and interstitial atoms. In this case, the number of defects formed due to a single creep of the step (by the length of the lattice constant) is equal to the number of atoms in the unit cell of the crystal. Thus, due to generation and motion of dislocations in crystals, along with proper dislocation electronic states (that is, those corresponding directly to the dislocation core), there also arise other states of defects. They are associated with some ordered nanostructures, namely the chains of point defects.

\section{Crystallographic orientation of dislocations around the micro-indentation}

Indented (110) surface. Development of the tangential and tetrahedral glide deformation on (110) surface in the vicinity of indenter fingerprints can be demonstrated by the scheme shown in Fig. 5. Tangential deformation arises at the surface of CdTe sample from the load of the indenter tip, and it develops plastic flow of the segment of the crystal lattice parallel to the surface in the inclined (111) and $(11 \overline{1})$ glide planes. Two sides of the moved segment represent (111) Cd (dark colour) and (111) Te (gray colour) planes. Intersection of such planes forms prisms which develop inside the crystals under an acute angle to the surface.

Indented (100) surface. This is the simplest rosette around the microindentation of a cubic crystal. It contains only two tangential systems of glide dislocations, where one system contains Cd dislocations, while another system is positioned under the right angle to the first one, and it contains Te dislocations. The geometry of a rosette around the microindentation on a (100) surface is sketched in Fig. 6.

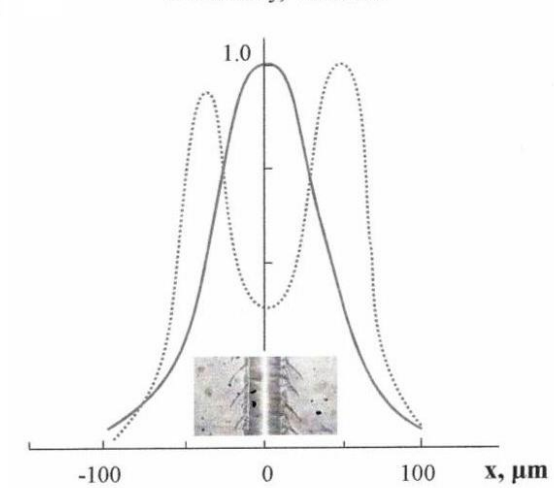

b

PL intensity, arb. units

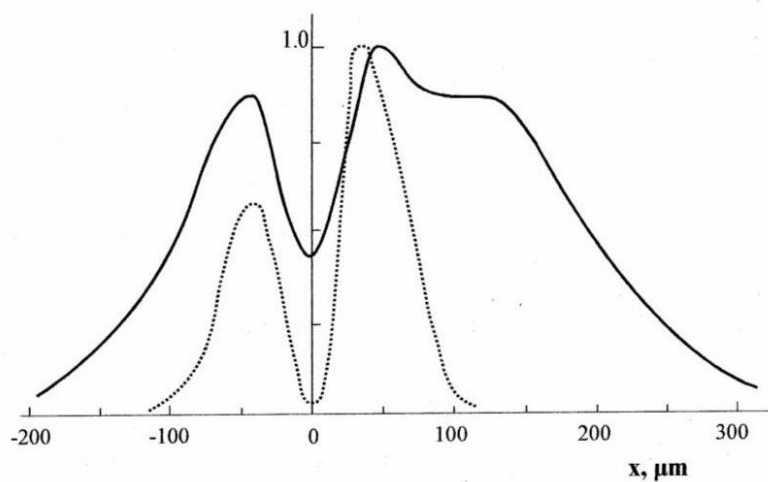

Fig. 4. Profiles of the spatial distribution of the PL radiation intensity in the 841-nm (solid curve) and 806-nm (dotted curve) bands on (001) face. Fig. 4a depicts the profiles for the $\langle 110\rangle$ direction, and Fig. $4 \mathrm{~b}$ for the $\langle 1 \overline{1} 0\rangle$ direction. The inset demonstrates an optical micro-photography of the deformed region in the $\langle 110\rangle$ direction on the (100) surface.

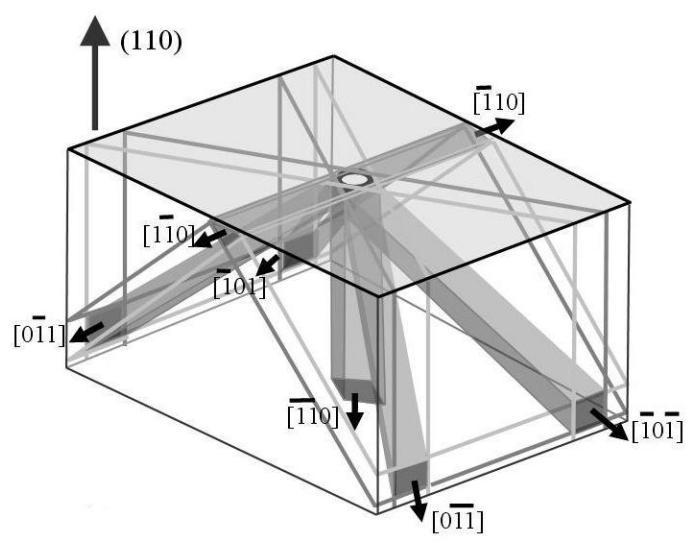

Fig. 5. Geometry of the rosette glide system around the microindentation on the (110) surface. Plastic flow takes place from the indented point shown by a circle placed in the center of the drawing in the inclined (111) and (11 $\overline{1})$ glide planes. From these planes $\mathrm{A}(\mathrm{g})$ - and $\mathrm{B}(\mathrm{g})$-dislocation branches arise.

\section{(C) 2014, V. Lashkaryov Institute of Semiconductor Physics, National Academy of Sciences of Ukraine}




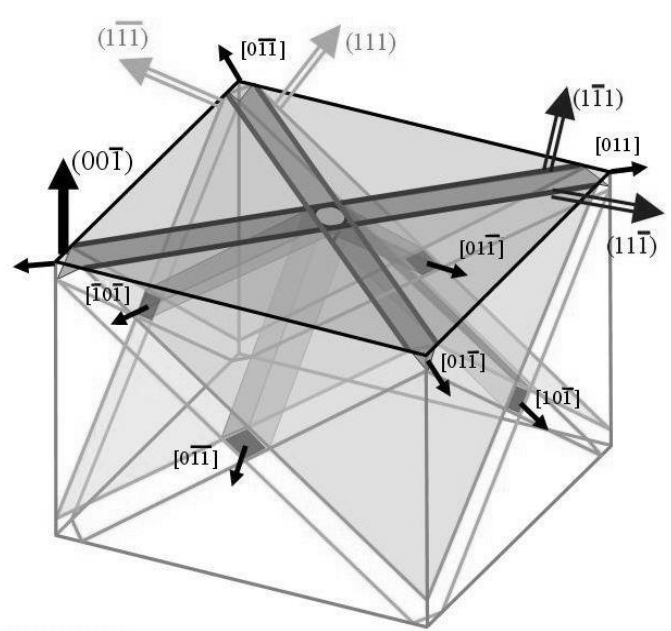

Fig. 6. Scheme of dislocation movements from the indented point on the (100) surface.

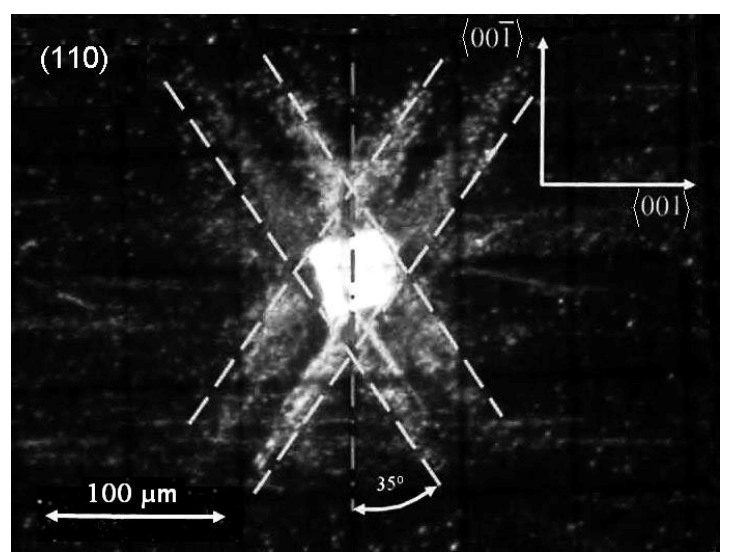

Fig. 7. Chemical etching rosette in the area of the indenter fingerprint on the (110) surface. White arrows show the Te dislocation branches, lines demonstrate crystallographic orientation as it is shown in Fig. 5.

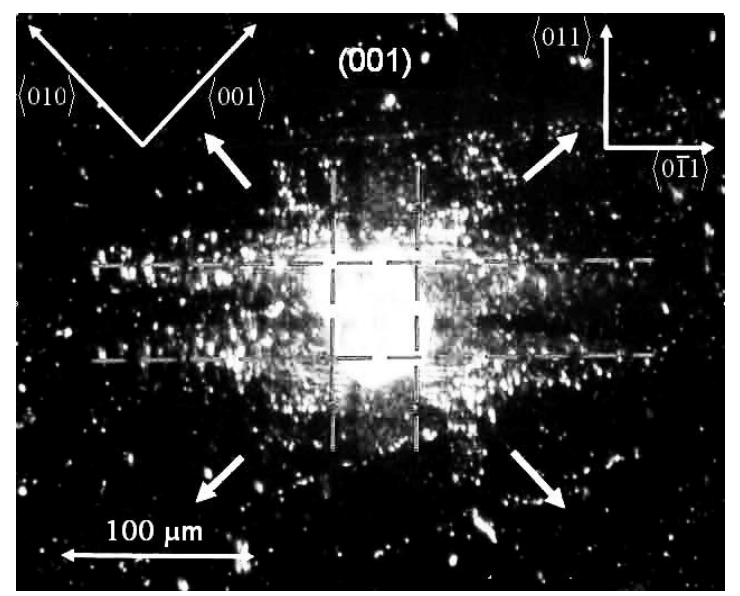

Fig. 8. Chemical etching rosette in the area of the indenter fingerprint on the (100) surface.

\section{Chemical etching rosette and luminescence at indentation of the (110) surface}

Experimental verification of the dislocation configuration as deduced from the model of surfaceparallel slip can be performed either by chemical etching, or using the luminescence mapping technique, and their coincidence with the sketched crystallographic configuration shown in Fig. 5 is demonstrated in Fig. 7.

The photo of the indented and etched (100) surface shown in Fig. 8 demonstrate similarity with the sketched in Fig. 6 distribution of dislocations in the (100) surface.

\section{Conclusions}

By comparing the various data, we are able to conclude that the "dislocation" emission is a characteristic emission in CdTe, as e.g., the well known so-called "edge" emission due to residual impurities. The "dislocation" emission has relatively narrow bands, which have different dependence on temperature and excitation intensity. It is reasonable to consider that these bands refer to the different types of deformation defects, namely, nanodefects.

\section{References}

1. N.I. Tarbaev and G.A. Shepelskii, One-dimensional structures formed by low-temperature slip of dislocations that act as sources of dislocation absorption and emission in II-VI semiconductor crystals // Semiconductors, 32(6), p. 580-586 (1998).

2. J. Schreiber, L. Yuring, H. Unievski, S. Hildebrant and H.S. Leipner, Recognition and distribution of $\mathrm{A}(\mathrm{g})$ and $\mathrm{B}(\mathrm{g})$ dislocations in indentation deformation zones on $\{111\}$ and $\{110\}$ surfaces of CdTe // Phys. Status Solidi (a), 171(1), p. 89-97 (1999).

3. V.D. Negrii, Yu.A. Osipian and N.V. Lomak, Dislocation structure and motion in CdS crystals // Phys. Status Solidi (a), 126(1), p. 49-61 (1991).

4. V. Babentsov, V. Boiko, R. Grill, J. Franc and G.A. Shepelskii, Point and nanoscale defects in $\mathrm{Cd}(\mathrm{S}, \mathrm{Se}, \mathrm{Te})$ crystals induced by plastic deformation // Phys. Status Solidi (c), 7(6), p. 14921494 (2010).

5. V. Babentsov, V. Boiko, G.A. Schepelskii, R.B. James, J. Franc, J. Prochazka and P. Hlidek, Photoluminescence and electric spectroscopy of dislocation-induced electronic levels in semiinsulated CdTe and CdZnTe // J. Lumin. 130, p. $1425-1430$ (2010).

6. V.N. Tomashik, Selective etching of chalcogenides of zinc, cadmium and mercury // Neorganicheskie materialy, 31(3), p. 313-317 (1995), in Russian. 